\title{
Foster care, syndemic health disparities and associations with HIV/STI diagnoses among young adult substance users
}

\author{
Mance E Buttram, ${ }^{1}$ Maria E Pagano, ${ }^{2}$ Steven P Kurtz ${ }^{1}$
}

${ }^{1}$ Center for Applied Research on Substance Use and Health Disparities, Nova Southeastern University, Fort Lauderdale, Florida, USA

2Department of Psychiatry, Division of Child Psychiatry, Case Western Reserve University, Cleveland, Ohio, USA

\section{Correspondence to}

Dr Mance E Buttram, Center for Applied Research on Substance Use and Health Disparities, Nova Southeastern University, Miami, FL 33138, USA; mance. buttram@nova.edu

Received 18 December 2017 Revised 1 June 2018 Accepted 7 July 2018 Published Online First 31 August 2018

\section{Check for updates}

(C) Author(s) (or their employer(s)) 2019. No commercial re-use. See rights and permissions. Published by BMJ.

To cite: Buttram ME,

Pagano ME, Kurtz SP.

Sex Transm Infect

2019:95:175-180

\section{ABSTRACT}

Objectives Foster care history is associated with many health and social problems, including sexual risk behaviours, HIV and other sexually transmitted infections (STIS). This cross-sectional study compares sexual risk behaviours among a sample of young adult substance users in Miami $(\mathrm{N}=602)$ with and without foster care histories.

Methods Participants completed a comprehensive assessment which included sections on foster care, sexual risk behaviours and related health and social problems. Logistic regression was used to examine the relationship between foster care history, associated syndemic vulnerabilities and increased likelihood of having a prior HIV/STI diagnosis.

Results Bivariate analyses indicated that foster care alumni were more likely to report a prior HIV/STI diagnosis, higher condomless sex frequencies, being high on alcohol or drugs during sex, sexual victimisation and a history of homelessness compared with other participants in the sample $(p<0.05)$. Multivariate analyses revealed that foster care history, sexual victimisation and group sex participation are associated with a prior HIV/STI diagnosis $(p<0.05)$. Group sex participation doubled the odds of a prior HIV/STI diagnosis for foster care alumni, compared with other participants $(p<0.5)$.

Conclusions This exploratory study identifies characteristics that distinguish foster care alumni from non-alumni and signals the need to better serve the sexual and related health needs of individuals with foster care histories. Clinicians and healthcare providers should provide foster care alumni with detailed HIV/STI risk reduction information and resources and services to address related syndemic vulnerabilities (eg, victimisation and homelessness).

\section{INTRODUCTION}

Estimates for 2015 indicate that more than 20000 young adults in the USA were emancipated from the foster care system. ${ }^{1}$ Youth and young adults ageing out of the foster care system often report limited educational attainment as well as a lack of adequate healthcare coverage, compared with other youth. ${ }^{23}$ This same group also has heightened vulnerability because of the presence of physical and mental health problems and inadequate knowledge about sexual risk reduction. ${ }^{4-6}$ Widespread negative experiences associated with foster care (eg, trauma, victimisation and unstable living situations) are associated with sexual risk behaviours. 478

Problems related to sexual behaviour are common among adolescents and young adults with foster care histories. Individuals ageing out of foster care may be at a higher propensity for engaging in sexual risk behaviours, ${ }^{6}$ including early initiation of sexual activity, ${ }^{59}$ increased number of sexual partners, ${ }^{10} 11$ and transactional sex. ${ }^{11} 12$ In addition, although sexually transmitted infection (STI) rates are similar for youth entering foster care and their peers without foster care involvement, STI rates significantly increase among youth during and after time in foster care, compared with their peers. ${ }^{6}$ Moreover, sexual risk behaviours are associated with HIV/STI infection among foster care alumni. $^{12} 13$

Although sexual risk behaviours among foster care alumni are well-documented, ${ }^{6}$ there is limited research on related syndemic vulnerabilities faced by this population, that puts them at greater risk for acquiring infectious disease. The syndemic production of health disparities-two or more risk factors that interact synergistically and contribute to an excess burden of disease-conceptualises health as the possession of physical, mental and social well-being that must be evaluated within a larger sociocultural context and has been employed in examinations of HIV/STI transmission risk, substance use, violence and other health and social disparities among many vulnerable populations. ${ }^{14}$ Foster care alumni also experience many syndemic health disparities, including risky sexual behaviours, drug use, mental health problems, neglect, victimisation and housing instability. $^{4-9} 111215$

Research on sexual risk behaviours and associated negative outcomes, including HIV/STI, is especially beneficial to clinicians and health and social service professionals working with foster care alumni. ${ }^{6}$ Such data are informative for healthcare providers who may fill a vital role in sexual education and HIV/STI testing for this population. ${ }^{16}$ Possessing a complete health profile and identifying specific characteristics and behaviours, including sexual risks and related syndemic health and social disparities, allows for sexual healthcare, prevention programmes, interventions as well as health and social services, to be tailored to vulnerable foster care alumni. ${ }^{17}$ 
Given that foster care alumni report many problems related to sexual behaviours and experience a multitude of syndemic vulnerabilities, examinations of sexual risk behaviours and related health and social problems among this population are needed. Among a sample of substance-using young adults, the present study has two primary aims: (1) to compare foster care alumni to non-alumni on measures of sexual behaviours and syndemic vulnerabilities and (2) building on the first aim, to examine associations of HIV/STI diagnosis among the sample, with a particular focus on foster care alumni. In addition, the analyses are designed to elucidate whether syndemic vulnerabilities differed between substance-using young adults with and without foster care histories.

\section{METHODS}

\section{Sample and recruitment}

Data are drawn from a behavioural substance use and sexual risk reduction intervention trial designed for substance-using young adults in the context of the electronic dance music (EDM) nightclub scene. A total of 602 participants completed comprehensive health and social risk assessments conducted between September 2011 and November 2015. Eligibility requirements included: (1) sex with an opposite-sex partner in the past 90 days (because of the design of the sexual risk reduction component); (2) ages 18-39; (3) use of one or more club drugs-cocaine, ecstasy, GHB (Gamma hydroxybutyrate), methamphetamine, ketamine, LSD (Lysergic acid diethylamide) —at least three times in the past 90 days; (4) non-medical use of one or more psychoactive medications at least once in the past 90 days and (5) regular attendance at large EDM nightclubs.

Participants were recruited through respondent-driven sampling (RDS). ${ }^{18}$ Initial respondents (seeds) were chosen for diversity in gender, ethnicity and age and recruited through outreach at nightclubs and existing contacts in the EDM culture. Each seed and subsequent study participant was provided with up to five recruitment coupons to give to other club drug users in their social network, with the understanding that they would earn $\$ 50$ for the recruitment of each additional eligible enrolled respondent. Although participants were not recruited at nightclubs, the clubs they reported patronising most often were large venues focused on the EDM experience.

Informed consent was obtained from participants prior to study participation. Interviews were conducted in private offices and lasted about $90 \mathrm{~min}$. Human subject protocols were approved by the university's Institutional Review Board.

\section{Measures}

The Global Appraisal of Individual Needs (GAIN, V.5.4) ${ }^{19}$ was the primary component of the standardised baseline assessments.

\section{Demographics and background}

Participants reported demographic characteristics (age, gender, race/ethnicity and education) and if they were ever placed in foster care. For foster care alumni, additional questions inquired about age of first placement, the relationship of the foster care placement(s) to the participant, the primary reason for the placement, length of time in foster care, and if the participant was ever removed from or ran away from a placement. Foster care alumni reported the quality of their foster care placement(s) on a 5-point Likert-type scale ranging from 'poor' to 'excellent'.

\section{HIV/STI history}

Participants reported current HIV status and lifetime diagnosis of any STI, including, herpes, chlamydia, syphilis, gonorrhoea, hepatitis B and human papilloma virus. All endorsements of any prior HIV/STI diagnoses were summed to construct the total number of HIV/STI incidences.

\section{Sexual behaviour}

Sexual behaviour questions included past 90 day counts of condomless vaginal and anal intercourse; these counts were combined and reported as the number of unprotected sexual encounters. Participants reported the age of first consensual sex (ie, not being pressured or forced to have sex against one's will) and if they had ever participated in group sex (defined as three or more people, including the participant). Transactional sex during the past 12 months was assessed by asking if the participant had purchased sex (ie, used drugs, gifts or money to purchase or get sex) or traded/sold sex (ie, have sex to get drugs, gifts or money).

\section{Substance use-related measures}

Substance use measures included past 90-day frequencies of use of a variety of substances including alcohol, marijuana, cocaine, ecstasy, methamphetamine, LSD, hallucinogens (eg, mushrooms, ketamine), GHB, heroin and the non-medical use of prescription (Rx) opioids, benzodiazepines and stimulants. Substance dependence symptoms were assessed using the seven Diagnostic and Statistical Manual of Mental Disorders, 4th edition (DSM-IV) symptoms during the past 90 days (eg, needing more alcohol/ drug to get the same effect; experiencing withdrawal symptoms). Being high for sex was assessed with the question, 'How often in the past 90 days were you "high" on either alcohol or drugs when you were having sex with another person?’ Responses were based on a five-point scale ranging from 'never' to 'almost all the time'. Given that all participants reported recent substance use, this measure was dichotomised into being 'high for sex almost all the time or more' versus not to identify those engaging in the riskiest substance use/sexual behaviours.

\section{Victimisation history}

Victimisation was assessed by affirmative responses to any of the following events: being attacked with a weapon or being beaten so as to cause bruises, cuts or broken bones (physical abuse); being forced to participate in sexual acts against one's will (sexual abuse) or being made to feel very bad about oneself or one's life (emotional abuse). Participants were also asked if any victimisation occurred prior to age 18 .

\section{Additional health and social problems}

The GAIN includes the General Mental Distress Scale, which includes past year DSM-IV symptom counts of somatisation (4 items, eg, sleep trouble; shortness of breath or lump in the throat), depression (9 items, eg, feeling sad, lonely or hopeless; feeling tired or having no energy) and anxiety (10 items, eg, feeling nervous, anxious or tense; unable to control worries). Homelessness was assessed by asking participants, 'When was the last time (if ever) that you considered yourself to be homeless or had to stay with someone else to avoid being homeless'. Responses were dichotomised into homelessness history versus not. 


\section{Analyses}

Statistical analyses were performed using the procedures CORR, FREQ and GENMOD of SAS V.9.3 (SAS, Cary, North Carolina, USA). Fisher's exact test for categorical variables and Wilcoxon Mann-Whitney rank-sum tests for continuous variables were used to test for differences between participants with and without foster care history. The relationship between foster care history, four associated syndemic vulnerabilities (group sex participation, being high on drugs/alcohol during sex, history of sexual victimisation, homelessness) and increased likelihood of having one or more HIV/STI diagnosis were examined in a logistic regression using the Poisson link function and Pearson correction for dispersion. Four interaction terms with foster care status were included to explore whether effects of syndemic vulnerabilities differed between foster care alumni and non-alumni. Characteristics that distinguished foster care status at $\mathrm{p}<0.10$ in univariate analyses were included as covariates, and age of first consensual sex was treated as an offset variable. Preliminary analyses suggested that linear modelling was adequate, and the logit model showed a good fit, $X^{2}=149.47, p=0.64$. Intracorrelations between explanatory variables did not exceed $r=0.2$ except between mental distress and substance dependency symptoms $(\mathrm{r}=0.3, \mathrm{p}<0.0001)$ and collinearity diagnostics indicated no problems. All p values were calculated with two-sided tests.

\section{RESULTS}

Of the 602 participants in the study, $8 \%(\mathrm{~N}=45)$ had a history of foster care placement that occurred at an average age of 9.09 years $(\mathrm{SD}=4.68$; range $=0-16)$. Approximately half $(52 \%)$ were placed with a non-related caregiver, $11 \%$ were placed with another biological family member and 36\% were placed with both. The most common primary reason for placement in foster care was parental alcohol and/or drug abuse (16\%), followed by physical abuse in the home (13\%), participant behavioural problems (13\%), parental death (11\%), sexual abuse in the home (9\%), parent/caregiver relinquishing the participant (9\%), parental incarceration (9\%), parental mental health problems (7\%), parental neglect (4\%), parental abandonment $(2 \%)$ or other (2\%). Participants lived an average of 4.27 years $(\mathrm{SD}=4.06$; range $=1-18)$ in foster care with an average of 8.78 placements $(\mathrm{SD}=18.54)$. Approximately half $(51 \%)$ were removed at least once from a foster care placement $(\mathrm{M}=2.38$; $\mathrm{SD}=5.97)$; participants were 11.72 years of age $(S D=3.53$; range $=2-16)$ when they were first removed from a foster care placement. More than half $(58 \%, 26 / 45)$ ran away at least once from a foster care placement $(M=13.69$ times; $S D=26.94)$, which first occurred when participants were 12 years of age $(S D=2.90$; range $=5-16)$. The average quality rating of foster care placements was 1.51 $(\mathrm{SD}=1.41$; range $=0-4)$.

Table 1 shows baseline characteristics of young adult substance users among participants with and without a foster care history With few exceptions, foster care alumni were similar to non-alumni in terms of age, victimisation history, substances used in the past 90 days and sexual risk indices; however, foster care alumni reported higher rates of sexual victimisation $(p<0.05)$, past 90 -day frequency of ecstasy use $(\mathrm{p}<0.001)$ condomless sex $(p<0.05)$, being high almost all the time during sex $(p<0.05)$ and have a history of homelessness $(p<0.05)$. Foster care alumni were more likely to be female $(\mathrm{p}<0.05)$, non-Hispanic and Black $(\mathrm{p}<0.05)$, with a trend towards more mental distress symptoms $(\mathrm{p}<0.10)$, substance dependence symptoms $(\mathrm{p}<0.10)$ and early age of initial consensual sex $(\mathrm{p}<0.10)$.
Of the 602 participants reported in this study, 22\% had a history of having one or more HIV/STIs, the most common of which were chlamydia (11\%), human papillomavirus (6\%), gonorrhoea $(5 \%)$, herpes (3\%), syphilis (2\%), Нepatitis B (1\%) and HIV (1\%). As shown in table 1, foster care alumni had significant higher rates of infectious disease history $(p<0.05)$ and higher count of infectious diseases $(p<0.001)$ than non-alumni. Specific types of infectious diseases that were higher among foster care alumni included: chlamydia ( $27 \%$ vs $\left.10 \%, \mathrm{X}^{2}=12.89, \mathrm{p}<0.001\right)$, gonorrhoea ( $13 \%$ vs $4 \%$, $\left.\mathrm{X}^{2}=7.16, \mathrm{p}<0.01\right)$, herpes $\left(9 \%\right.$ vs $\left.3 \%, \mathrm{X}^{2}=5.23, \mathrm{p}<0.05\right)$ and syphilis ( $7 \%$ vs $\left.1 \%, \mathrm{X}^{2}=7.71, \mathrm{p}<0.01\right)$.

Table 2 shows the relationship between foster care, associated syndemic vulnerabilities and their interaction in relation to prior HIV/STI diagnosis, controlling for six characteristics that distinguished foster care alumni at baseline (gender, race/ethnicity, condomless sex frequencies, days using ecstasy, mental distress, substance dependence symptoms). Results showed significant main effects for foster care history $(p<0.05)$, sexual victimisation $(p<0.001)$ and group sex participation $(p<0.05)$; the predicted odds of prior HIV/STI diagnosis was $69 \%$ higher for foster care alumni $(\mathrm{OR}=1.69), 76 \%$ higher for group sex participants $(\mathrm{OR}=1.76)$ and tripled $(\mathrm{OR}=3.18)$ for victims of sexual abuse. A significant interaction between foster care and group sex participation history $(p<0.05)$ showed that the negative effects of group sex participation on risk of infectious disease doubled for foster care alumni $(\mathrm{OR}=2.78)$. Of these covariates, significant main effects were found only for gender $(p<0.05)$; the predicted odds of prior HIV/STI diagnosis was $43 \%$ higher for females $(\mathrm{OR}=1.43)$.

\section{DISCUSSION}

This study examined sexual behaviours and related syndemic vulnerabilities associated with prior HIV/STI diagnosis, with an emphasis on associations with foster care history among a sample of substance-using young adults. The findings indicate that, compared with individuals without foster care histories, foster care alumni are more likely to report a prior HIV/STI diagnosis and more HIV/STI incidences as well as multiple health and social problems, including sexual and substance use risk behaviours, victimisation and homelessness. The increased likelihood of experiencing HIV/STI diagnosis among individuals with foster care histories is worrisome and the co-occurrence of multiple health and social problems likely exacerbates the vulnerabilities already experienced by this population.

In the multivariate model predicting one or more prior HIV/ STI diagnosis, foster care alumni had 69\% higher odds than individuals without foster care histories; women, individuals with sexual victimisation histories and those who have participated in group sex also had higher odds of reporting an HIV/STI diagnosis. The model also included interaction terms to examine whether syndemic vulnerabilities differed among individuals with and without foster care histories; results indicate that while group sex participation significantly increases the risk of HIV/ STI diagnosis for all participants, the effects are much stronger among foster care alumni.

Importantly, this study demonstrates risks related to group sex among behaviourally heterosexual young adults. Group sex events are those in which individuals have sex with more than one partner during a single sexual encounter. During group sex, bodily fluids may be shared through condomless sex and the use of hands, toys and/or condoms with multiple partners in succession. ${ }^{2021}$ Participants from multiple 


\begin{tabular}{|c|c|c|c|c|c|}
\hline Variable & & Total $(n=602,100 \%)$ & Foster care $(n=45,8 \%)$ & No foster care $(n=557,92 \%)$ & Test ( $p$ values) \\
\hline \multicolumn{6}{|l|}{ Demographics } \\
\hline Age & (M/SD) & $25.8(5.42)$ & $26.67(5.29)$ & $25.72(5.43)$ & $\mathrm{F}=1.26$ \\
\hline \multicolumn{6}{|l|}{ Gender } \\
\hline Female & (\% yes) & $259(43 \%)$ & $26(58 \%)$ & $233(42 \%)$ & $\chi^{2}=4.31^{*}$ \\
\hline \multicolumn{6}{|l|}{ Race } \\
\hline Hispanic & ( $\%$ yes) & $385(64 \%)$ & $20(44 \%)$ & $365(65 \%)$ & $\chi^{2}=19.10^{* * *}$ \\
\hline Black & (\% yes) & $138(23 \%)$ & $22(49 \%)$ & $116(21 \%)$ & \\
\hline White & (\% yes) & $65(11 \%)$ & $3(7 \%)$ & $62(11 \%)$ & \\
\hline Other & (\% yes) & $14(2 \%)$ & $0(0 \%)$ & $14(5 \%)$ & \\
\hline \multicolumn{6}{|l|}{ Education } \\
\hline Years & (M/SD) & $12.76(1.52)$ & $12.48(1.58)$ & $12.78(1.52)$ & $\mathrm{F}=1.52$ \\
\hline \multicolumn{6}{|l|}{ HIV/STI history } \\
\hline Number of HIVISTI incidences & (M/SD) & $0.54(1.22)$ & $1.13(1.89)$ & $0.49(1.13)$ & $\mathrm{F}=11.91 * * *$ \\
\hline One or more HIV/STI diagnoses & (\% yes) & $134(22 \%)$ & $16(36 \%)$ & $118(21 \%)$ & $\chi^{2}=5.00^{*}$ \\
\hline \multicolumn{6}{|l|}{ Sexual behaviours } \\
\hline Age of first consensual sex & (M/SD) & $15.03(2.18)$ & $14.42(2.36)$ & $15.08(2.17)$ & $\mathrm{F}=3.31^{\mathrm{t}}$ \\
\hline Group sex participation history & (\% yes) & $244(41 \%)$ & $23(51 \%)$ & $221(40 \%)$ & $\chi^{2}=2.26$ \\
\hline Purchased sex & (\% yes) & $65(11 \%)$ & $7(16 \%)$ & $58(10 \%)$ & $\chi^{2}=1.14$ \\
\hline Traded/sold sex & (\% yes) & $74(12 \%)$ & $7(16 \%)$ & $67(12 \%)$ & $\chi^{2}=0.48$ \\
\hline No. sexual partnerst & (M/SD) & $5.32(8.71)$ & $5.98(9.51)$ & $5.27(8.65)$ & $\mathrm{F}=0.28$ \\
\hline Condomless sex frequenciest & (M/SD) & $28.75(49.90)$ & $42.82(48.45)$ & $27.61(50.00)$ & $\mathrm{F}=3.87^{*}$ \\
\hline \multicolumn{6}{|l|}{ Substance use-related measures } \\
\hline \multicolumn{6}{|l|}{ Past 90-day frequencies: } \\
\hline Alcohol & (M/SD) & $45.79(25.53)$ & $51.33(26.40)$ & $45.35(25.43)$ & $\mathrm{F}=2.29$ \\
\hline Marijuana & (M/SD) & $60.12(33.11)$ & $63.11(31.36)$ & $59.87(33.26)$ & $\mathrm{F}=0.40$ \\
\hline Cocaine & (M/SD) & $30.09(26.14)$ & $36.84(31.39)$ & $29.54(25.63)$ & $F=3.26$ \\
\hline Ecstasy & (M/SD) & $28.89(23.37)$ & $40.53(29.14)$ & $27.95(22.61)$ & $\mathrm{F}=12.29^{* * *}$ \\
\hline Methamphetamine & (M/SD) & $3.53(12.18)$ & $4.56(14.95)$ & $3.44(11.94)$ & $\mathrm{F}=0.35$ \\
\hline LSD & (M/SD) & $4.98(11.14)$ & $4.53(9.72)$ & $5.02(11.26)$ & $\mathrm{F}=0.08$ \\
\hline Other hallucinogens & (M/SD) & $5.78(12.44)$ & $4.80(13.22)$ & $5.86(12.38)$ & $\mathrm{F}=0.30$ \\
\hline GHB & (M/SD) & $2.08(9.25)$ & $0.87(3.68)$ & $2.17(9.55)$ & $\mathrm{F}=0.83$ \\
\hline Heroin & (M/SD) & $6.62(19.85)$ & $7.13(20.55)$ & $6.58(19.81)$ & $\mathrm{F}=0.03$ \\
\hline Rx opioid & $(\mathrm{M} / \mathrm{SD})$ & $29.86(28.67)$ & $36.40(31.16)$ & $29.33(28.43)$ & $F=2.54$ \\
\hline Rx benzodiazepine & (M/SD) & $31.70(29.53)$ & $37.33(27.76)$ & $31.25(29.65)$ & $\mathrm{F}=1.77$ \\
\hline Rx stimulant & (M/SD) & $10.15(18.90)$ & $12.51(21.05)$ & $9.96(18.72)$ & $\mathrm{F}=0.76$ \\
\hline Substance dependence symptoms $\ddagger \S$ & (M/SD) & $3.67(2.39)$ & $4.24(2.26)$ & $3.62(2.40)$ & $\mathrm{F}=2.84^{\mathrm{t}}$ \\
\hline High during sex almost all the timet, & (\% yes) & $218(36 \%)$ & $22(50 \%)$ & $196(35 \%)$ & $\chi^{2}=3.80^{*}$ \\
\hline \multicolumn{6}{|l|}{ Victimisation history } \\
\hline Any victimisation & ( $\%$ yes) & $447(74 \%)$ & $36(80 \%)$ & $411(74 \%)$ & $\chi^{2}=0.84$ \\
\hline If yes, victimised as a minor & (\% yes) & $351(58 \%)$ & $29(64 \%)$ & $322(58 \%)$ & $\chi^{2}=0.73$ \\
\hline Physical victimisation & (\% yes) & $264(44 \%)$ & $23(51 \%)$ & $241(43 \%)$ & $\chi^{2}=1.04$ \\
\hline Sexual victimisationt† & (\% yes) & $110(18 \%)$ & $14(31 \%)$ & $96(17 \%)$ & $\chi 2=5.31^{*}$ \\
\hline Emotional victimisationt† & (\% yes) & $322(54 \%)$ & $29(64 \%)$ & $293(53 \%)$ & $\chi^{2}=2.27$ \\
\hline \multicolumn{6}{|l|}{ Additional health and social problems } \\
\hline Mental distress symptoms $\ddagger \S$ & $(\mathrm{M} / \mathrm{SD})$ & $8.04(6.73)$ & $9.76(7.93)$ & $7.91(6.61)$ & $F=3.16^{t}$ \\
\hline Homelessness history & (\% yes) & $299(50 \%)$ & $35(78 \%)$ & $264(47 \%)$ & $\chi^{2}=15.32 * * * *$ \\
\hline
\end{tabular}

${ }^{*} \mathrm{p}<0.05 ;{ }^{* *} \mathrm{p}<0.01 ;{ }^{* * *} \mathrm{p}<0.001 ;{ }^{* * * *} \mathrm{p}<0.0001 ; \mathrm{t}=\mathrm{p}<.10$.

tPast 90 days.

$\ddagger$ Past year.

$\S n=1$ no data.

१n $\mathrm{n}=3$ no data

$\dagger+n=2$ no data.

GHB, Gamma hydroxybutyrate; LSD, Lysergic acid diethylamide; STI, sexually transmitted infection.

disparate populations (eg, people who use drugs, men who have sex with men, sex buyers or exchangers) may attend the same group sex events, facilitating the transmission of HIV/
STI across group boundaries; HIV/STI discordance among group sex participants is common and the potential for HIV/ STI transmission is high. ${ }^{20}$ However, foster care alumni are 
Table 2 Poisson regression predicting likelihood of one or more HIV/STI diagnoses among substance-using young adults (N=602) $\dagger$

\begin{tabular}{|c|c|c|c|c|c|}
\hline & B & SE & $x^{2}$ & OR & $95 \% \mathrm{Cl}$ \\
\hline \multicolumn{6}{|l|}{ Demographics } \\
\hline Foster care history & 0.53 & 0.30 & $3.12^{*}$ & 1.69 & -0.06 to 1.11 \\
\hline Gender (ref=female) & -0.36 & 0.18 & $4.16^{*}$ & 0.70 & -0.71 to -0.01 \\
\hline \multicolumn{6}{|l|}{ Race (ref=Black) } \\
\hline Hispanic & -0.69 & 0.61 & 1.26 & 0.50 & -1.89 to 0.51 \\
\hline White & -0.21 & 0.31 & & & \\
\hline Other & 0.42 & 0.31 & & & \\
\hline \multicolumn{6}{|l|}{ Sexual behaviours } \\
\hline Group sex participation history & 0.56 & 0.28 & $4.01^{*}$ & 1.76 & 0.01 to 1.12 \\
\hline Condomless sex frequencies $\ddagger$ & 0.00 & 0.00 & 0.18 & 1.00 & -0.01 to 0.00 \\
\hline \multicolumn{6}{|l|}{ Substance use-related measures } \\
\hline Days ecstasy use $\ddagger$ & -0.00 & 0.01 & 0.78 & 0.99 & --0.02 to 0.01 \\
\hline Substance dependence symptoms§ & -0.06 & 0.08 & 0.59 & 0.94 & -0.22 to 0.10 \\
\hline High during sex almost all the time & 0.11 & 0.27 & 0.16 & 1.11 & -0.42 to 0.63 \\
\hline \multicolumn{6}{|l|}{ Victimisation history } \\
\hline Sexual victimisation & 1.16 & 0.31 & $14.29^{* * *}$ & 3.18 & 0.56 to 1.76 \\
\hline \multicolumn{6}{|l|}{ Additional health and social problems } \\
\hline Mental distress symptoms§ & 0.01 & 0.03 & 0.15 & 1.01 & -0.04 to 0.07 \\
\hline Homelessness history & -0.24 & 0.30 & 0.67 & 0.79 & -0.82 to 0.34 \\
\hline \multicolumn{6}{|l|}{ Interactions } \\
\hline Foster care $\times$ group sex participation & -0.60 & 0.30 & $3.94^{*}$ & 0.83 & -1.20 to -0.01 \\
\hline Foster care $\times$ sexual victimisation & -0.18 & 0.28 & 0.44 & 0.55 & -0.73 to 0.36 \\
\hline Foster care $\times$ homelessness & 0.43 & 0.29 & 2.20 & 1.54 & -0.14 to 1.00 \\
\hline Foster care $\times$ high during sex almost all the time & -0.07 & 0.27 & 0.07 & 0.93 & -0.59 to 0.45 \\
\hline
\end{tabular}

${ }^{*} \mathrm{p}<0.05 ;{ }^{* *} \mathrm{p}<0.01 ;{ }^{* * *} \mathrm{p}<0.001$.

$+22 \%$ (134/602) with one or more HIV/STI diagnoses;

$\ddagger$ Past 90 days.

$\S$ Past year.

STI, sexually transmitted infection.

especially vulnerable because of their widespread experiences with health and social problems, many of which are associated with increased sexual risk behaviour. ${ }^{47811} 12$ Taken together, the risks related to group sex participation and increased likelihood of HIV/STI incidence would add to the problems faced by youth and young adults with foster care histories and would likely exacerbate existing vulnerabilities.

The findings indicate a segment of young adult substance users who experience a unique set of factors that must be addressed by clinicians and healthcare providers. Multiple placements and unstable home lives mean that youth in foster care are liable to miss sexual health education in schools; however, these youths generally visit a primary healthcare provider on each new foster placement, which presents an opportunity to provide comprehensive sexual risk reduction education in a safe and confidential setting. ${ }^{16}$ Detailed sexual risk reduction information, including HIV/STI transmission risks during group sex, delivered to youth in foster care is needed and primary care visits may be the only place in which this vulnerable population receives such information. Considering the syndemic nature of many of these problems, ${ }^{14}$ future HIV/STI diagnosis may be mitigated if clinicians and healthcare providers screen for additional health and social problems, including substance use, victimisation and homelessness and actively connect foster care alumni to additional resources and services.

In addition, opportunities for sexual risk reduction also exist outside of healthcare settings. Including adjunct sexual health modules to existing resilience-focused and caregiver-based interventions, ${ }^{22}$ and mentoring programmes, ${ }^{23}$ would likely be impactful for vulnerable foster care alumni. Furthermore, research demonstrates that parental support and involvement is associated with lower sexual risk behaviours. ${ }^{24} 25$ Such findings suggest that sexual health education delivered by foster care parents would also be beneficial. Creating opportunities for sexual health educators and clinicians to provide sexual health training and support for foster care parents to encourage these practices is warranted.

This study has some limitations worth noting. The ability to generalise the findings to other foster care alumni and/or substance-using young adults is limited by the study eligibility requirements. Additionally, these analyses did not account for the diversity of foster care experiences (eg, length of time; quality of care) and the number of foster care alumni in the sample was small. Future research is needed to replicate study findings with a larger sample of foster care alumni. In addition, data are based on self-report and some STIs may not have ever been diagnosed, potentially leading to underreporting of STI diagnoses as well as other socially undesirable behaviours.

This study identifies characteristics that distinguish foster care alumni from non-alumni and signals the need to better serve the sexual health and related needs of individuals with foster care histories. The results cohere with existing research indicating that youth and young adults with foster care histories report more sexual risk behaviours than their peers ${ }^{6}$ and contributes to the limited literature describing risks related to group sex. These data suggest that clinicians and healthcare providers should 
address a full range of sexual behaviours and provide foster care alumni with comprehensive sex education and resources to address related health and social problems.

\section{Key messages}

- This study compared sexual risk behaviours among a sample of young adult substance users in Miami with and without foster care histories.

- Foster care alumni were more likely to report being high on alcohol/drugs during sex, higher condomless sex frequencies and prior sexually transmitted infection (STI) diagnosis than other participants.

- Group sex significantly increases the risk of HIV/STI diagnosis for all participants, but the effects are much stronger among foster care alumni.

- Clinicians should provide foster care alumni with HIVISTI risk reduction information and resources to address related syndemic vulnerabilities.

\section{Handling editor Jackie A Cassell}

Contributors MEB conceptualised the study, contributed to data analyses and developed a draft of the manuscript. MEP performed statistical analyses and contributed to the interpretation of findings. SPK served as Principal Investigator of the primary study, contributed to study conceptualisation and to the interpretation of findings. All authors participated approved the final version of the manuscript.

Funding This research was supported by DHHS Grant Number 5 R01 DA019048 from the National Institute on Drug Abuse.

Disclaimer The content is solely the responsibility of the author and does not necessarily represent the official views of the National Institute on Drug Abuse or the National Institutes of Health.

Competing interests None declared.

Patient consent Not required.

Ethics approval Nova Southeastern University IRB.

Provenance and peer review Not commissioned; externally peer reviewed.

Data sharing statement The data are not available.

\section{REFERENCES}

1 U.S. Department of Health and Human Services Administration for Children and Families, Administration on Children, Youth and Families, Children's Bureau, 2016. The AFCARS Report - Preliminary Estimates for FY 2015 as of June 2016. Available from: https://www.acf.hhs.gov/sites/default/files/cb/afcarsreport23.pdf [Accessed accessed 11 April 2017].

2 Day A, Dworsky A, Fogarty K, et al. An examination of post-secondary retention and graduation among foster care youth enrolled in a four-year university. Child Youth Serv Rev 2011;33:2335-41.

3 Dworsky A, Ahrens K, Courtney M. Health insurance coverage and use of family planning services among current and former foster youth: implications of the health care reform law. J Health Polit Policy Law 2013;38:421-39.
4 Ahrens KR, McCarty C, Simoni J, et al. Psychosocial pathways to sexually transmitted infection risk among youth transitioning out of foster care: evidence from a longitudinal cohort study. J Adolesc Health 2013;53:478-85.

5 Leslie LK, James S, Monn A, et al. Health-risk behaviors in young adolescents in the child welfare system. J Adolesc Health 2010;47:26-34.

6 Ramseyer Winter V, Brandon-Friedman RA, Ely GE. Sexual health behaviors and outcomes among current and former foster youth: A review of the literature. Child Youth Serv Rev 2016;64:1-14.

7 Dworsky A, Napolitano L, Courtney M. Homelessness during the transition from foster care to adulthood. Am J Public Health 2013:103(Suppl 2):S318-S323.

8 Gonzalez-Blanks A, Yates TM. Sexual risk-taking among recently emancipated female foster youth: Sexual trauma and failed family reunification experiences. I Res Adolesc 2016;26:819-29.

9 Stott T. Placement instability and risky behaviors of youth aging out of foster care. Child and Adolescent Social Work Journal 2012;29:61-83.

10 Fowler PJ, Motley D, Zhang J, et al. Adolescent maltreatment in the child welfare system and developmental patterns of sexual risk behaviors. Child Maltreat 2015:20:50-60.

11 Wilson E, Casanueva C, Smith KR, et al. Risk of early sexual initiation and pregnancy among youth reported to the child welfare system. Child Welfare 2014:93:127-47.

12 Ahrens KR, Katon W, McCarty C, et al. Association between childhood sexual abuse and transactional sex in youth aging out of foster care. Child Abuse Negl 2012;36:75-80.

13 Surratt HL, Kurtz SP. Foster care history and HIV infection among drug-using African American female sex workers. AIDS Behav 2012;16:982-9.

14 Ahrens KR, Richardson LP, Courtney ME, et al. Laboratory-diagnosed sexually transmitted infections in former foster youth compared with peers. Pediatrics 2010;126:e97-e103.

15 Singer M. Introduction to syndemics: a critical systems approach to public and community health. San Francisco: Jossey-Bass, 2009.

16 Thompson RG, Auslander WF. Substance use and mental health problems as predictors of HIV sexual risk behaviors among adolescents in foster care. Health Soc Work 2011:36:33-43.

17 Hudson AL. Where do youth in foster care receive information about preventing unplanned pregnancy and sexually transmitted infections? J Pediatr Nurs 2012;27:443-50.

18 Kools S, Paul SM, Jones R, et al. Health profiles of adolescents in foster care. J Pediatr Nurs 2013;28:213-22.

19 Heckathorn DD. Respondent-driven sampling: a new approach to the study of hidden populations. Soc Probl 1997;44:174-99.

20 Dennis ML. Global Appraisal of Individual Needs. Version 5.4. Bloomington, IL: Chestnut Health Systems, 2006.

21 Friedman SR, Bolyard M, Khan M, et al. Group sex events and HIV/STI risk in an urban network. J Acquir Immune Defic Syndr 2008;49:440-6.

22 Friedman SR, Mateu-Gelabert P, Sandoval M. Group sex events amongst non-gay drug users: an understudied risk environment. Int I Drug Policy 2011;22:1-8.

23 Flannery JE, Beauchamp KG, Fisher PA. The role of social buffering on chronic disruptions in quality of care: evidence from caregiver-based interventions in foster children. Soc Neurosci 2017;12:86-91.

24 Taussig HN, Culhane SE, Garrido E, et al. RCT of a mentoring and skills group program: placement and permanency outcomes for foster youth. Pediatrics 2012;130:e33-e39.

25 Taussig HN. Risk behaviors in maltreated youth placed in foster care: a longitudinal study of protective and vulnerability factors. Child Abuse Negl 2002;26:1179-99.

26 Taussig HN, Talmi A. Ethnic differences in risk behaviors and related psychosocial variables among a cohort of maltreated adolescents in foster care. Child Maltreat 2001;6:180-92. 